\title{
Organizational Decision Support Systems
}

\author{
Paulo Garrido 1 \\ pgarrido@dei.uminho.pt \\ 1 University of Minho, 4800 Guimaraes, Portugal
}

\begin{abstract}
This paper presents a notion for developing Organizational Decision Support Systems (ODSS). The lens used is to see ODSS as a specialization of Social Decision Support Systems (SDSS) and a generalization of Group Decision Support Systems (GDSS). A unit decision process can be raised by any person in a organizational whole, the organization itself or some sub-organization. The decision process can use a given formalism coherent with the idea that as the contributions from people in the decision process expands, so does the space of available perceptions (P), the space of available actions (A), strategies $(\mathrm{S})$, and options $(\mathrm{O})$, the space of available criteria $(\mathrm{C})$ of valuation of options $(\mathrm{V})$, leading to decision. Atomic decision processes link in the global decision process of the organization. Potential benefits, shortcomings and needed technologies are indicated as well as prospects of development.
\end{abstract}

Keywords: organizational decision support systems; social decision support systems; collective intelligence; decision support systems; collective decision-making.

\section{Introduction}

Information and communication systems substitute and enlarge human capabilities. One striking aspect of such enlargement has been the development of automatic control devices that substitute people in decision making. Another, the development of information systems for decision support, covering several aspects of decision processes (Power, 2002).

Turoff et al. (2002) introduced the notion of a Social Decision Support System (SDSS) as a type of information system, with first objective "to facilitate the integration of diverse views into a growing knowledge base". Other related approaches include Rodriguez and Steinbock (2004), Rodriguez et al. (2007). One can enlarge the concept of a SDSS to include the support and taking of some conceivable decision by some conceivable population. Iandoli et al. (2007) report field tests for an actual application to climate change.

In such generality of possible applications, one stands out of special interest: organizational decision. Among social groups, organizations distinguish themselves by the stable recurrence of people and common functional goals. Being widespread is one of the reasons that make one suspect that their success is of crucial importance to the well-being of human societies. It is natural to suspect that properties of the decision process each uses are of crucial important to its success. The specialization of the notion of SDSS, to fit the characteristics of organizations, appears as an evident step to get into the notion of Organizational Decision Support Systems (ODSS). An account of previous research in the subject may be found in Garrido and Faria (2008). 
Obvious advantage can be gained in seeing such a move as companion to a dual move of generalization of Group Decision Support Systems (GDSS) (Huber, 1982) (Turoff and Hiltz, 1982).

The notion of ODSS presented here, being as general as possible, acknowledges primarily the role of conversations among people in organizational decision processes. If conversations do not occur, organizational decisions are taken in an absolute individual fashion or are taken by automatic devices. These no-conversation modes can have their usefulness, but it is hard to deny that, from some level up, conversations among people are necessary for good decisions, if not only for decisions, and that people's practice expresses such necessity.

One can see conversations as having another role besides being a tool for deciding: often they spot the need to trigger other decision processes. This is the same to say that often decisions originate in conversations about decisions. In this way, decisions connect forming chains, trees or loops.

This notion of ODSS sees decision processes in an organization as constituting, through coexistence and integration, the global conversational decision process (GCDP) of the organization, henceforth global decision process (GDP), for short. ODSS should help to better the GDP by making it explicit, through the mapping and interconnecting of unit conversational decision processes (UDPs) and suggesting its analysis.

An ODSS should be able to connect to other decision support and information systems of the organization in order to ease and speed the flow of information among them. In this way, the ODSS will become a hub organizing decision, and the flows of information to and from decision.

Given the above characteristics posited, I think the organizations that can benefit first from this notion of ODSS are those whose leaders and, desirably, the decision-makers and all participants:

i) recognize that conversations among people are a necessary and effective component in a vast class of required or possible decisions (usually the more important ones), and

ii) want to explore and exploit the potential benefits of supporting such decisions in an integrated way along the organization based on information systems.

A framework for the support of UDPs is presented in Section 2. In Section 3, the framework is extended for the integration of UDPs in the GDP of the organization.

Section 4 reviews potential benefits and shortcomings and identifies issues for design and development not addressed in this paper.

\section{Unit conversation decision processes}

I consider here a unit conversation decision process (UCDP), or unit decision process (UDP) for short, to correspond to a well defined organizational whole or set of people. Inside an organization there can be many such wholes as the organization itself or their sub-organizations (divisions, departments, project groups, for instance). A UDP should usually output a stream of decisions either internally or externally motivated. The people in the whole to which the UDP corresponds are supposed to be potential contributors to the decisions in the stream.

Inside a whole some people will be in charge of taking decisions, some will not. For short, I will use the term "decisor" for someone in charge of taking decisions. Depending on the decisions, at one extreme only one people in a whole will be a decisor, at the other extreme all the people will be decisors. Also depending on the decision protocol for decisions, some will be taken individually and some will be taken collectively. In any case, a period of conversation potentially enlarged to all the people in the whole is supposed to happen before the decision is taken, and one of the core functions of an ODSS is to facilitate and support this period. 
Why would one take a decision with support from a conversation? In other words, which benefits can a person taking a decision get from receiving inputs or advice from other people? This appears a good question for any people in the whole when s/he must take a decision, either being the only to take the decision for the whole, or participating in the collective decision taking protocol. If all the people in the whole are called to a collective decision, they still do not escape to make an individual decision, the collective decision being some composition of individual decisions.

The obvious answer to the questions above is that receiving inputs from other people may help in arriving to a decision that generates outcomes better for the individual decisor and for the whole than would be the case if the decision would be taken alone. This is a "make individual sense" condition. For the condition to be maintained in the long term it is also necessary that the people in the whole do perceive the sequence of decisions taken as generating outcomes better for them. If that will not be the case, the decisor will not find willingness of the people to give inputs and his or her potential gain will become impossible. To have " $n$ heads think better than one" it must be clear for each of the $n$ that there is a personal and a collective gain in the thinking. The personal gain may fail to happen at each decision but should be positive along the sequence of decisions the person perceives, as far as the person is able or interested in perceiving. I think that the more this conscience and its consequences are taken in account by decisors (and by non decisors), the more an ODSS can be useful to the people in an organization.

\subsection{Formalism for supporting unit decision processes}

A more refined and insightful answer to the questions above can be obtained if we take a formal analysis of the decision process and use this analysis to conceive the support an ODSS should make available to decision processes with conversations. The following formalism is a classical one, with elements from Simon (1976), Newell and Simon (1972). It is presented as a possible example to base implementations.

Let us consider a person that must take a decision alone. To decide, the person disposes of a set of perceptions $P_{1}$. This set encompasses, necessarily, perceptions about the environment or state of affairs, $S_{1}$ that does not meet some reference criteria $R_{1}$. If the criteria reduce to one only criterion, one will write $r_{1}$. The issue of taking a decision is raised in first place by the perception of the criteria not being satisfied, and the judgment that something could be done or not done with respect to it. This creates a goal $g_{1}$, which could be:

- Assessing the solvability of the problem of satisfying the criterion to be met;

- Given the solvability, accessing the relevance or urgency of doing so in the frame of other decisions of the whole;

- Given both above, to select from different courses of action or options, that which expectably will lead to satisfying the criterion.

In any case, the person will perceive or conceive of a set of available actions $A_{1}$, from which a subset must be chosen, forming an option. This formulation allows defining the set of options $O_{1}$, as all possible combinations of taking or not taking the actions in $A_{1}$, the power set of $A_{1}$.

Using a set of criteria $C_{1}$, the person will valuate the consequences of each option, creating a set of valuations, $V_{1}=C_{1}\left(O_{1}\right)$ from which the decision should follow. Note that $C_{1}$ should include $R_{1}$.

By asking other $n-1$ people to contribute through conversations, the person can potentially enlarge the set of perceptions s/he disposes to a set $P_{C}$. Formally, one can view such set as the union of perceptions of each person:

$$
P_{C}=P_{1} \cup P_{2} \cup \ldots \cup P_{n}
$$


There will be a potential gain in terms of a better decision for the decisor if $P_{C}$ strictly includes $P_{1}: P_{C} \supset P_{1}$. Otherwise, the decisor gets no new inputs and her or his decision cannot be different from taking the decision alone. In this case, s/he (and the organizational whole) may incur effectively a loss by the costs inherent in the conversation from which the contributions arose. However, in this case, a subjective gain may be considered either enlarged confidence on the decision or a warning on the decision capabilities of the whole.

Enlarging the set of perceptions may be the result of enlarging the set of perceptions of the state of affairs, the set of available actions, and the set of criteria. As with $P_{C}$, one can view formally such enlargement as unions:

$$
\begin{aligned}
& S_{C}=S_{1} \cup S_{2} \cup \ldots \cup S_{n} \\
& A_{C}=A_{1} \cup A_{2} \cup \ldots \cup A_{n} \\
& C_{C}=C_{1} \cup C_{2} \cup \ldots \cup C_{n}
\end{aligned}
$$

Because of (1.2) the set of options and the set of valuations also may grow:

$$
\begin{aligned}
& O_{C}=\operatorname{powerset}\left(A_{C}\right) \\
& V_{C}=C_{C}\left(O_{C}\right)
\end{aligned}
$$

The analysis and formalism above suggest that an ODSS should support UDPs through collecting and registering contributions in a way that makes evident the statement of the issue or goal for initiating the decision process, the information about the state of affairs, the possible actions and the criteria, consequent options and valuations from each contribution.

\subsection{Flow of the decision process}

Let us imagine roughly how a UDP with conversation would go. Say that an issue has been raised. This means that, at least, a description $S_{1}$ of a state of affairs and an applied reference criteria $R_{1}$ providing evidence for / establishing the necessity of deciding doing something else about it (other than what has been done) have been presented through the ODSS. Possibly, actions to take, options and their valuations may have been presented, all through adequate forms. Then, other people can contribute enlarging the first presentation of the issue. After some time the decisor seems appropriate, for example, because no real enlarging on any of the sets is observed or just because of time constraints, s/he takes the decision.

Let us note the following. It is recognizable that the decision process implies a flow, and it seems a good idea to require that ODSS provide states and transitions between states to formalize the flow. Say that an issue has been raised by a non-decisor. It may make sense that it is recognized as worth examining to enter the decision process. If recognized it may also make sense to prioritize it. States of raised, recognized, prioritized are possible descriptions. When the decision is taken, the issue may be said to be decided. Upon getting confirmation that the decision worked it may be said closed. Transitions between states should be the responsibility of the decisor in charge. This implies that a decisor may pose an issue in the recognized, or prioritized state from the beginning. It can even make sense that a decisor poses the issue as decided.

Any potential benefit from conversation in decision process comes with the cost of involving more people in the process, which means that they must spend time and energy to think or research about their aspects and the contributions of others, and to transmit to the system the results of their thinking or research. Because of inherent costs, decisors always face the problem of establishing a trade-off in allocating resources to the decision process. Stating an issue as decided (without conversation) may be the best course of action in many cases. The notion of ODSS presented here targets enlarging behavioral possibilities of people inside an organization, not restricting them. 


\subsection{Polling and voting mechanisms}

Supporting decisors through conversation or any other contributions does not entail that the decision itself should be taken by other people than the decisor. If i) a decisor consistently arrives to good decisions in the sense that people in the organizational whole perceive that in the long term decisions are beneficial to them and ii) people in the organizational whole understand that their contributions are necessary for good decisions, then it is expectable that they contribute without conditioning their contributions to these being accepted or the decision being mandatorily collective - that is being arrived at by some voting process.

However, the existence of polling or voting mechanisms at each organizational whole level in a ODSS is a must, or at least, a distinctive advantage. On one hand, a decisor may want to know what the result of a poll is on some aspect of an issue or on the whole issue - and eventually decide accordingly. On the other hand, voting may be mandatory for some decisions and it may be useful to have such possibility in the ODSS.

\section{The global decision process}

In an organization, many decision processes occur recurrently and simultaneously. The decision processes are dependent and, often, strongly inter-related. I envisage the function of an ODSS as giving support to the global conversational decision process of the organization, global decision process (GDP) for short. It is the union and composition of unit decision processes.

In this paper, I consider four issues in conceiving such support. Sub-organizations and the address space of each person, the linking of decisions, the explicit map of decision processes and implementation aspects.

\subsection{Sub-organizations and the address space}

Organizations have sub-organizations. These have been referred above as wholes of people to which decisions respect. It is clear that one cannot expect much usefulness from raising issues to people if they are unrelated to their interests - or if the people cannot contribute to their decision.

Mathematically speaking, if an organization has a set of $m$ people, it is possible to conceive of $2^{m}$ subsets of people. In practice a few of all these possible subsets will correspond to suborganizations or wholes - the organization itself being one of them and the largest. Some subsets are included in other, for example, a department may be included in a division, some not, for example, a project group with people from several departments. Some are more stable, some more transitory.

It follows that it seems a good idea that an ODSS supports the definition of sub-organizations and the attribution of people to them. In each sub-organization, it is to expect that decision processes recur, occur simultaneously and are inter-related as they are in the organization as a whole. For this, I mean to require that the ODSS will allow for mapping the configuration of suborganizations inside the organization, each sub-organization being supported in their decision processes as such.

Along a set theoretical reasoning, a person necessarily belongs to more than one subset of people or sub-organization. Say that s/he belongs to sub-organization $X$. Then it also belongs to any sub-organization $Y$ which set of people includes the set of people of $X$, in particular s/he belongs to the universe of people of the organization. Furthermore, it can belong to other sets for which the $Y$ inclusion relation does not hold.

Should a person be allowed to raise decision issues to any subset of people or suborganization? In other words, as information overload should be minimized, which should be the 
address space of each person to pose decision issues? A seemingly natural criterion seems to define such possible address space as the set of all subsets of people in the organization to which the person belongs. Say that a person belongs to department $X$, of division $Y$ of organization $U$. Then she can pose decisions either to $X$, or to $Y$ or to $U$. Such arrangement expectably will preserve relevance of issues posed, participation of people, and promote clear thinking about decisions.

\subsection{Relating and linking decision processes}

Linking and relating decision processes in the global decision process of an organization can be seen to happen at a whole level (inside a sub-organization and independently of others) as well at a multi-whole level (say from a division to a department and then up again).

Two possible relations of decision processes, which gave naturally rise to linking, are sequencing and nesting.

I will say that decision process $D_{1}$ will be in sequence with $D_{2}$ if $D_{2}$ has been triggered by $D_{1}$, but the completion or closing of $D_{1}$ does not depend on $D_{2}$. I will say that decision process $D_{1}$ will nest $D_{2}$ if $D_{2}$ has been triggered by $D_{1}$, and the completion or closing of $D_{1}$ does depend on the completion of $D_{2}$.

If an ODSS allows for the tagging of decision processes in terms of their sequencing and nesting relations (and the associated implementation of decisions) it will be possible to develop visualizations and analysis of the unfolding of the global decision process, a seemingly most useful possibility.

Other possibilities of relating and linking are of interest. Decision processes can be classified by type. Assuming that an ODSS will keep the records of most (if not any) decision processes in the organization, such information can be explored to look for similar decision problems, best solutions, or to develop facilitators for people to raise or pose issues.

\subsection{Explicitly mapping decision processes}

The conceptual architecture for ODSS presented raises the possibility of explicitly mapping the global decision process and their constituent processes in several ways, for example through textual or graphical visualizations.

I think this is a most advantageous possibility to consider, explore and exploit. Such mapping would allow for a clear conscience among people in the organization (or the organizational whole) of the issues at stake, their relative importance, the existence of decision processes and how these could be changed and made more efficient.

In this view, one can view ODSS in a learning perspective: they are devices through which people learn to decide in better ways either alone or collectively.

\subsection{Implementation aspects}

A straightforward implementation of a ODSS could use a web or intranet server configuration. Depending on the size of the organization, the ODSS could be partitioned through several machines or software instances. Linking of the instances becomes an important facility as well as connecting the ODSS to other information systems in the organization.

Such an implementation configuration would allow support for synchronous (actual or virtual meetings) or asynchronous interactions among people and between people and the ODSS. Asynchronous interactions have interesting sparing time prospects as eliminating (unnecessary) meetings or facilitating meeting preparation. 


\section{Review, conclusions and perspectives}

This paper has presented a notion for Organizational Decision Support Systems (ODSS). Several ideas converge in this notion. If one considers the ubiquitous role and practice of conversations in many decision processes, and the character of top-level of these ${ }^{1}$, then it seems a good idea to explore the possibility of substantially improving decisions quality in organizations by taking the "support of conversations" as the main "tool" to arrive at decisions.

The notion presented sees an ODSS as mapping in itself the configuration of suborganizations of the organization. It becomes natural that the interface with users be framed through such a map, each user addressing or relating with decision processes occurring in the suborganizations or organizational wholes s/he belongs to.

The notion also supposes that some formalism for decision processes is to base the interaction among users, or the collecting and communication of users' contributions. Users may be decisors or non-decisors, but, in principle, all are deemed as potential contributors to decisions, including raising issues to be decided.

To exploit further the potential advantages of an ODSS, this should allow perceiving decision processes as state transition flows, with associated devices to enable polling and voting on issues, linking and classifying decision processes. This raises the possibility of mapping through the ODSS the global decision process of the organization, making the interaction of such an information system a space for organizational learning.

\subsection{Potential benefits and pitfalls}

At the individual decision level, the basic fact giving a potential gain for decisors and the organizational wholes is that an ODSS can ease or facilitate:

- To perceive potential relevant issues for decision as any member can raise an issue.

- To get an enlarged perception about the issue to be decided upon, that may at least translate in a better decision under the current frame of thinking. In an even more favorable outcome, it may translate in a better frame of thinking to consider the issue.

One may expect that the greater the diversity or variety (Asbhy, 1956) of contributions, the more probable is the potential gain. Assuring such diversity - in particular, preventing groupthink (Wikipedia, 2009) and "organizational silence" (Morrison and Milliken, 2000) - is a major concern. In an ODSS, anonymous contributions should be enabled in a way that people trust.

On the other hand, some issues raised may be irrelevant or the perception created by the conversation process may not enlarge the perception of the issue, or give rise to a better decision that an issue's presenter had in mind when presenting it. Human time costs incurred in the decision process would be then unproductive. This problem in se can also occur without using an ODSS, and using an ODSS could inflate it. This potential pitfall prompts for three necessary steps of understanding:

- That an ODSS as a support system aims at greater automation of the decision processes but it cannot substitute people thinking and judgment.

- That using an ODSS entails a period of individual and organizational learning.

- That the general principles for an ODSS stated above can be implemented in many flexible ways desirably matched to the reality of the people in the organization and their current processes.

In particular, the possibility that all the people contribute to decision does not entail that all people decide. Additionally, it also does not entail that people are obliged to contribute with extra effort for the organization.

\footnotetext{
${ }^{1}$ Being top-level means they are not conducted by machines nor are approached in a individual basis by decisors.
} 
At the global decision level, the basic fact giving a potential gain for the people in the organization is the possibility of mapping the global decision process of the organization in very flexible ways, able to support meaningful communication to and from people.

\subsection{Future research}

Any decision process has a cost in itself, and going for an ODSS as described will have inherent costs, both in investment and operating costs. Profitability hinges on synergistic factors some of them I will indicate as being:

- Improved decisions quality;

- Improved motivation and satisfaction of people;

- Improved efficiency of the global and the local decisions processes of the organization;

- Organizational learning.

All these in turn should translate into more added value per capita produced by the organization. To get estimates of the value of the increase is a difficult task, given that a not-yettried concept is being proposed and given that locally unpredictable, relevant gains to expect, may come from non-linear effects of interaction among people an ODSS may enable. However, logically deriving bounds from a set of reasonable assumptions may give a first map of possibilities.

Having established the interest in implementing an ODSS, the following questions to address relate to how one must design the ODSS so people using it start to produce better decision processes with minimal learning. Design should also aim i) smooth integration in the culture and established ways of interaction among people and ii) effective integration in the global information system of the organization. In requiring smooth integration in the culture, one does not mean that this culture will not change as a result of the organization adopting an ODSS.

\section{Acknowledgements}

I am grateful to Nelson Faria for cooperation and support. The funding of FCT (Fundação a Ciência e Tecnologia) is gently acknowledged.

\section{References}

Ashby, W. R. (1956). An Introduction to Cybernetics. Chapman \& Hall, London.

Garrido, P. and Faria, N. (2008), Design of a social decision support system for organizations, in Boaventura, J. Proceedings of Controlo 2008 - The Eight Portuguese Conference on Automatic Control, (Vila Real, Portugal, 2008).

Huber, G. P. (1982). Group decision support systems as aids in the use of structured group management techniques. In Transactions of the $2^{\text {nd }}$ International Conference on Decision Support Systems, pp. 96-103.

Iandoli, L., Klein, M. and Zollo, G. (2007) Can We Exploit Collective Intelligence for Collaborative Deliberation? The Case of the Climate Change Collaboratorium. MIT Sloan Research Paper No. 4675-08. Available at SSRN: http://ssrn.com/abstract=1084069

Morrison, E. W. and Milliken, F. J. (2000). Organizational Silence: A Barrier to Change and Development in a Pluralistic World. The Academy of Management Review, 25(4), pp. 706725. 
Newell, A., and Simon, H. A. (1972). Human Problem Solving. Englewood Cliffs, NJ: PrenticeHall.

Power, D. J. (2002). Decision Support Systems: concepts and resources for managers. Quorum Books, Westport, Conn.

Rodriguez, M., and D. Steinbock (2004). A Social Network for Societal-Scale Decision-Making Systems. In: North American Association for Computational Social and Organizational Science Conference Proceedings 2004. Also available at: http://arxiv.org/abs/cs.CY/0412047

Rodriguez, M. A.; Steinbock, D. J.; Watkins, J. H.; Gershenson, C.; Bollen,J.; Grey, V.; and deGraf, B. (2007). "Smartocracy: Social Networks for Collective Decision Making". In HICSS '07: Proceedings of the 40th Annual Hawaii International Conference on System Sciences, page 90, Washington DC, USA. IEEE Computer Society.

Simon, H. A. (1976). Administrative Behavior. The Free Press, New York,USA.

Turoff, M. and Hiltz, S. (1982). Computer support for group versus individual decisions. IEEE Transactions on Communications, COM-30:1, pp. 82-90.

Turoff, M., S. R. Hiltz, H.-K. Cho, Z. Li and Y. Wang (2002). Social Decision Support Systems. In: Proceedings of the 35th Hawaii International Conference on System Sciences. Also available at: http://www.hicss.hawaii.edu/HICSS_35/HICSSpapers/PDFdocuments/CLCSC03.pdf

Wikipedia (2009) Groupthink. http://en.wikipedia.org/wiki/Groupthink 